\title{
ANALYTICAL SOLUTIONS FOR VIBRATION OF SIMPLY SUPPORTED NONLOCAL NANOBEAMS WITH AN AXIAL FORCE
}

\author{
C. $\mathrm{LI}^{*, \dagger}$, C. W. $\mathrm{LIM}^{\dagger, \ddagger}$, J. L. YU* and Q. C. ZENG ${ }^{\dagger}$ \\ *Department of Modern Mechanics \\ University of Science and Technology of China \\ Hefei, Anhui 230026, P. R. China \\ ${ }^{\dagger}$ Department of Building and Construction \\ City University of Hong Kong, Tat Chee Avenue \\ Kowloon, Hong Kong SAR, P. R. China \\ †bccwlim@cityu.edu.hk
}

Received 19 February 2010

Accepted 29 April 2010

\begin{abstract}
This paper presents exact, analytical solutions for the transverse vibration of simply supported nanobeams subjected to an initial axial force based on nonlocal elasticity theory. Classical continuum theory is inherently size independent while nonlocal elasticity exhibits size dependence. The latter has significant effects on bending moment, which results in a conceptually different definition of a new effective nonlocal bending moment with respect to the corresponding classical bending moment. A sixth-order partial differential governing equation is subsequently obtained. The effects of nonlocal nanoscale on the vibration frequencies and mode shapes are considered and analytical solutions are solved. Effects of the nonlocal nanoscale and dimensionless axial force including axial tension and axial compression on the first three mode frequencies are presented and discussed. It is found that the nonlocal nanoscale induces higher natural frequencies and stiffness of the nano structures.
\end{abstract}

Keywords: Frequency; nanobeam; nonlocal elasticity; nonlocal bending moment; vibration.

\section{Notations}

a Internal nanoscale characteristic length

$A$ Cross-sectional area of the nanobeam

$B$ Width of the nanobeam

C Constant

\$Corresponding author. 
$e_{0}$ Material constant

$E$ Young's modulus

$H$ Thickness of the nanobeam

I Second moment of area

$L$ Length of the nanobeam

$M, \bar{M}$ Dimensional and dimensionless nonlocal bending moment

$M_{\mathrm{ef}}, \bar{M}_{\mathrm{ef}} \quad$ Dimensional and dimensionless effective nonlocal bending moment

$n$ Order number

$P, \bar{P} \quad$ Dimensional and dimensionless initial axial compression

$P_{\text {cr }}, \bar{P}_{\text {cr }} \quad$ Dimensional and dimensionless critical axial compression

$q_{n}$ Temporal function

$Q$ Shear force

$t, \bar{t}$ Dimensional and dimensionless temporal coordinate

$T, \bar{T}$ Dimensional and dimensionless initial axial tension

$w, \bar{w}$ Dimensional and dimensionless transverse displacement

$x, \bar{x}$ Dimensional and dimensionless axial coordinate

y Transverse coordinate

$\rho$ Mass density

$\tau$ Dimensionless nonlocal nanoscale

$\psi_{n} \quad$ Vibration mode function

$\Omega_{n}, \omega_{n}$ Dimensional and dimensionless vibration frequency

$\Omega_{n}^{\mathrm{c}} \quad$ Classical dimensional vibration frequency

\section{Introduction}

Nanotubes and nanobeams play key roles in many engineering devices at nanometer scale, such as micro- or nanoelectromechanical systems (MEMS or NEMS). ${ }^{1,2}$ Although classical theories of linear and nonlinear vibration of strings and beams at macroscales are well established, the vibration behavior of structures at nanoscale which is significantly size dependent is far from being well understood. In an effort to extend the classical theory for size dependent nanomechanics, the vibration of a nanobeam is investigated herein based on the nonlocal elastic stress theory.

Owing to the scarcity of research in vibration of nanobeams subject to an axial tension or motion, an account of research works at the macroscale and nanoscale are described here. Simpson ${ }^{3}$ addressed the vibration frequency and mode function of axially moving beams without initial tension and with clamped conditions at two ends. The train-induced vibration of a parabolic tied-arch beam via an analytical approach was investigated by Yau. ${ }^{4}$ Al-Bedoor and Khulief ${ }^{5}$ studied axially moving beams with slow velocity and different support conditions. Transverse nonlinear parametric vibration of an axially accelerating viscoelastc string was introduced by Chen et $a l .{ }^{6}$ and the method of multiple scales was applied directly to the nonlinear 
partial differential equation to investigate the principal resonance. Oz et al. ${ }^{7}$ applied the method of multiple scales to study dynamic stability of an axially accelerating beam with small bending stiffness. For a nanobeam, Guo and Zhao ${ }^{8}$ presented a theoretical model to investigate the size-dependent bending elastic properties with influence of surface effects. Lim and $\mathrm{Wang}^{9}$ constructed a complete and asymptotic representation of nanobeams with nonlocal stress via an exact variational principle approach.

The nonlocal beam models received increasing interest in the past few years. Nonlocal continuum theories regard the stress state at a point as a function of the strain states of all points in the body while the classical continuum mechanics assumes the stress state at a given point is dependent uniquely on the strain state at that same point. The static deformation of nanobeams based on a simplified nonlocal model was obtained firstly by Eringen ${ }^{10,11}$ and Kroner. ${ }^{12}$ The application of nonlocal continuum theories to nanotechnology was revived recently by Peddieson et al. ${ }^{13}$ and they paid particular attention to cantilever nanobeams which were often used as actuators in small-scale systems.

The nonlocal theory of elasticity was used to study applications in nanomechanics including lattice dispersion of elastic waves, wave propagation in composites, dislocation mechanics, fracture mechanics, surface tension fluids, etc. ${ }^{14-20}$ Of all the nanostructures, the mechanical behaviors of nanotubes and nanobeams have been most widely investigated. ${ }^{21-28}$ For example, Challamel and Wang ${ }^{27}$ considered the small length scale effect for a nonlocal cantilever beam while Zhang et al. ${ }^{28}$ applied such a model and considered higher-order boundary conditions related to a variational principle for generalized loading situations, including vibration and buckling. There exist repeated conclusions that nonlocal nanoscale results in lower vibration frequency and such conclusions should be carefully revisited because they imply that nonlocal size effects cause nanostructural stiffness to decrease. ${ }^{13-16,18,19,21-28}$ Some authors believe that the consequence of reduced or increased structural stiffness is due to different choices of higher-order boundary conditions which appeared in the nonlocal nanobeam model. However, such opinions are still being investigated and could not be concluded in the current state.

With respect to the conclusion and belief above, it was discovered recently by $\mathrm{Lim}^{29-32}$ that the reverse trend of nonlocal nanoscale effect which induces increased nanostructural stiffness should take place. In this paper, a new approach to the nonlocal model is presented and the nonlocal effects of a nanobeam subject to an initial axial force are investigated. First, the effective nonlocal bending moment is established as an infinite series via the energy variation principle. Considering only the first nonlocal term which carries the most significant nonlocal effect, a sixth-order partial differential equation and six higher-order, nonclassical boundary conditions are derived. ${ }^{29-40}$ Subsequently, an analytical method is developed to derive exact solutions for free vibration frequencies and mode shapes which satisfy the higherorder, nonlocal classical boundary conditions. The effects of initial axial force and 
nonlocal stress on vibration behavior of nanobeams are discussed and some numerical examples of a silicon nanobeam are presented to show the differences due to the presence of a nonlocal nanoscale.

\section{Nonlocal Equations of Motion}

Consider a nanobeam with an initial axial tension $T$ at the ends and length $L$. The force equilibrium diagram of an element of the nanobeam is illustrated in Fig. 1.

In Fig. $1 M_{\text {ef }}$, to be defined in due course, is the effective nonlocal bending moment ${ }^{29-32}$ according to the nonlocal elasticity theory, $T$ the internal axial force, $Q$ the shear force, $x$ the axial coordinate, and $y$ the transverse coordinate. Considering linear vibration with only small deformation, the dynamic equation of motion for the element can be obtained based on Newton's second law of motion and the moment equilibrium condition as

$$
\frac{\partial^{2} M_{\mathrm{ef}}}{\partial x^{2}}+T \frac{\partial^{2} w}{\partial x^{2}}-\rho A \frac{\partial^{2} w}{\partial t^{2}}=0
$$

where $\rho$ is the material mass density, $A$ the cross-sectional area of nanobeam, and $w$ the transverse displacement and the effective nonlocal bending moment ${ }^{29-32}$

$$
M_{\mathrm{ef}}=M-2 \sum_{n=1}^{\infty}\left(e_{0} a\right)^{2 n} \frac{\partial^{2 n} M}{\partial x^{2 n}} \quad(n=0,1,2, \ldots),
$$

in which the nonlocal bending moment

$$
M=\left(e_{0} a\right)^{2} \frac{\partial^{2} M}{\partial x^{2}}-E I \frac{\partial^{2} w}{\partial x^{2}},
$$

and $E I$ is the flexural rigidity. Two other quantities $e_{0}$ and $a$ represent the nonlocal effects are, respectively, a constant dependent on material and an internal characteristic length nanoscale. ${ }^{11}$ Terms of higher-order partial derivatives in Eq. (1) are ignored and the internal axial force is assumed to equal to the initial tension $T$.

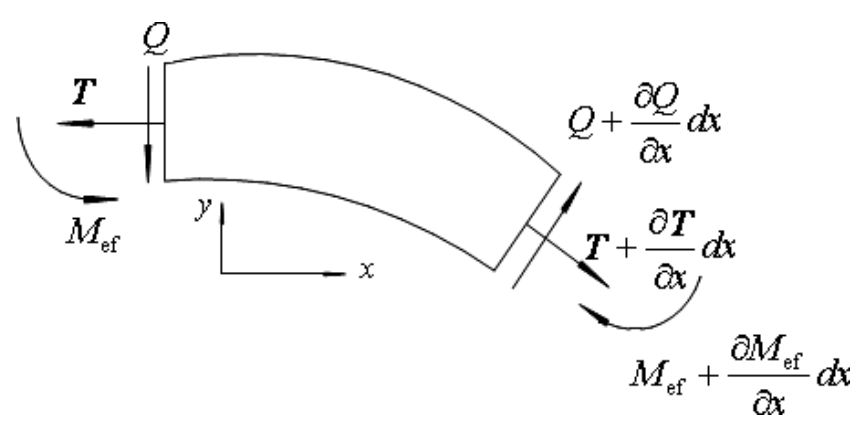

Fig. 1. Force and moment equilibrium for an element of the nanobeam. 
Considering $n=1$ for $M_{\mathrm{ef}}$, in which the most significant nonlocal effect is retained, and further substituting into Eq. (1) yields

$$
\frac{\partial^{2} M}{\partial x^{2}}-2\left(e_{0} a\right)^{2} \frac{\partial^{4} M}{\partial x^{4}}+T \frac{\partial^{2} w}{\partial x^{2}}-\rho A \frac{\partial^{2} w}{\partial t^{2}}=0 .
$$

From Eqs. (3) and (4), the governing equation of motion for a nanobeam subjected to an initial axial tension can be derived as

$$
\rho A \frac{\partial^{2} w}{\partial t^{2}}-T \frac{\partial^{2} w}{\partial x^{2}}-\left(e_{0} a\right)^{2}\left(2 E I \frac{\partial^{6} w}{\partial x^{6}}+\rho A \frac{\partial^{4} w}{\partial x^{2} \partial t^{2}}-T \frac{\partial^{4} w}{\partial x^{4}}\right)=-E I \frac{\partial^{4} w}{\partial x^{4}} .
$$

The relation between nonlocal bending moment and transverse displacement is also obtained according to Eqs. (3) and (4) as

$$
M=\left(e_{0} a\right)^{2} \rho A \frac{\partial^{2} w}{\partial t^{2}}+\left[E I-T\left(e_{0} a\right)^{2}\right] \frac{\partial^{2} w}{\partial x^{2}}+2 E I\left(e_{0} a\right)^{2} \frac{\partial^{4} w}{\partial x^{4}} .
$$

From Eqs. (2) and (6a), one gets

$$
\begin{aligned}
M_{\mathrm{ef}}= & \left(e_{0} a\right)^{2} \rho A \frac{\partial^{2} w}{\partial t^{2}}+\left[E I-T\left(e_{0} a\right)^{2}\right] \frac{\partial^{2} w}{\partial x^{2}}+2 E I\left(e_{0} a\right)^{2} \frac{\partial^{4} w}{\partial x^{4}} \\
& -2\left(e_{0} a\right)^{2}\left\{\left(e_{0} a\right)^{2} \rho A \frac{\partial^{4} w}{\partial x^{2} \partial t^{2}}+\left[E I-T\left(e_{0} a\right)^{2}\right] \frac{\partial^{4} w}{\partial x^{4}}+2 E I\left(e_{0} a\right)^{2} \frac{\partial^{6} w}{\partial x^{6}}\right\}
\end{aligned}
$$

where $n=1$ is adopted in Eq. (2). Introducing the following dimensionless parameters and variables

$$
\bar{x}=\frac{x}{L}, \quad \bar{w}=\frac{w}{L}, \quad \bar{t}=t \sqrt{\frac{E I}{\rho A L^{4}}}, \quad \tau=\frac{e_{0} a}{L}, \quad \bar{T}=\frac{T L^{2}}{E I}, \quad \bar{M}=\frac{M L}{E I} .
$$

Equations (5), (6a), and (6b) can be expressed in dimensionless terms as

$$
\begin{gathered}
\frac{\partial^{2} \bar{w}}{\partial \bar{t}^{2}}-\bar{T} \frac{\partial^{2} \bar{w}}{\partial \bar{x}^{2}}+\frac{\partial^{4} \bar{w}}{\partial \bar{x}^{4}}-\tau^{2} \frac{\partial^{4} \bar{w}}{\partial \bar{x}^{2} \partial \bar{t}^{2}}+\bar{T} \tau^{2} \frac{\partial^{4} \bar{w}}{\partial \bar{x}^{4}}-2 \tau^{2} \frac{\partial^{6} \bar{w}}{\partial \bar{x} 6}=0 \\
\bar{M}=\tau^{2} \frac{\partial^{2} \bar{w}}{\partial \bar{t}^{2}}+\left(1-\bar{T} \tau^{2}\right) \frac{\partial^{2} \bar{w}}{\partial \bar{x}^{2}}+2 \tau^{2} \frac{\partial^{4} \bar{w}}{\partial \bar{x}^{4}} \\
\bar{M}_{\mathrm{ef}}=\tau^{2} \frac{\partial^{2} \bar{w}}{\partial \bar{t}^{2}}+\left(1-\bar{T} \tau^{2}\right) \frac{\partial^{2} \bar{w}}{\partial \bar{x}^{2}}+2 \tau^{2} \frac{\partial^{4} \bar{w}}{\partial \bar{x}^{4}} \\
-2 \tau^{2}\left[\tau^{2} \frac{\partial^{4} \bar{w}}{\partial \bar{x}^{2} \partial \bar{t}^{2}}+\left(1-\bar{T} \tau^{2}\right) \frac{\partial^{4} \bar{w}}{\partial \bar{x}^{4}}+2 \tau^{2} \frac{\partial^{6} \bar{w}}{\partial \bar{x} 6}\right],
\end{gathered}
$$

where the non dimensional nanoscale parameter $\tau<0.2$ for most materials.

For linear free vibration of a nanobeam, the method of variables separation can be applied. The solution for Eq. (8) can be assumed as

$$
\bar{w}_{n}(\bar{x}, \bar{t})=C \psi_{n}(\bar{x}) q_{n}(\bar{t})
$$


where $C$ is an arbitrary constant, $\psi_{n}(\bar{x})$ is the vibration mode function, $q_{n}(\bar{t})$ the temporal function with respect to $\bar{t}$ and $n=1,2,3, \ldots$ denotes the mode number. By substituting Eq. (10) into Eq. (8), one obtains an ordinary differential equation as

$$
\begin{gathered}
\frac{d^{2} q}{d \bar{t}^{2}}+\omega_{n}^{2} q=0 \\
-\omega_{n}^{2} \psi_{n}+\left(\tau^{2} \omega_{n}^{2}-\bar{T}\right) \frac{d^{2} \psi_{n}}{d \bar{x}^{2}}+\left(\bar{T} \tau^{2}+1\right) \frac{d^{4} \psi_{n}}{d \bar{x}^{4}}-2 \tau^{2} \frac{d^{6} \psi_{n}}{d \bar{x} 6}=0,
\end{gathered}
$$

where $\omega_{n}$ is dimensionless vibration frequency. Obviously, $q_{n}(\bar{t})=e^{i \omega_{n} \bar{t}}$ is obtained from Eq. (11a), where $\omega_{n}=\Omega_{n} \sqrt{\frac{\rho A L^{4}}{E I}}$ is the dimensionless natural frequency, in which $\Omega_{n}$ is the physical dimensional frequency.

\section{Analysis, Results, and Discussion}

To illustrate the effects of nonlocal nanoscale $\tau$ and dimensionless axial tension $\bar{T}$ on the vibration behavior of a nanobeam, a simply supported nanobeam is considered. The higher-order, nonclassical boundary conditions corresponding to the generalized stress and displacement conditions are ${ }^{29-32}$

$$
\begin{array}{ll}
\bar{M}_{\mathrm{ef}}(0, \bar{t})=0 & \bar{M}_{\mathrm{ef}}(1, \bar{t})=0 \\
\bar{w}(0, \bar{t})=0 & \bar{w}(1, \bar{t})=0 \\
\frac{\partial^{2} \bar{w}}{\partial \bar{x}^{2}}(0, \bar{t})=0 & \frac{\partial^{2} \bar{w}}{\partial \bar{x}^{2}}(1, \bar{t})=0
\end{array}
$$

These conditions are regarded as nonclassical because of the existence of higher-order boundary conditions. According to the classical beam theory, the first two boundary conditions are zero classical bending moment and zero deflection. However, the last boundary condition, which is the point of inflexion, is not required in the classical theory. Furthermore, according to the classical beam theory, the first and the last boundary conditions are the same because the zero classical bending moment condition is equivalent to the condition for point of inflexion. However, in the nonlocal beam theory, $\bar{M}_{\mathrm{ef}} \neq \bar{M} \neq \partial^{2} \bar{w} / \partial \bar{x}^{2}$ as indicated in Eqs. (9a) and (9b).

The substitution of Eqs. (9b) and (10) into Eq. (12) and simplifying the expressions yields

$$
\begin{array}{ll}
\bar{T} \frac{d^{4} \psi_{n}}{d \bar{x}^{4}}(0)-2 \frac{d^{6} \psi_{n}}{d \bar{x} 6}(0)=0 & \bar{T} \frac{d^{4} \psi_{n}}{d \bar{x}^{4}}(1)-2 \frac{d^{6} \psi_{n}}{d \bar{x} 6}(1)=0 \\
\psi_{n}(0)=0 & \psi_{n}(1)=0 \\
\frac{d^{2} \psi_{n}}{d \bar{x}^{2}}(0)=0 & \frac{d^{2} \psi_{n}}{d \bar{x}^{2}}(1)=0 .
\end{array}
$$

For a sixth-order partial differential equation and six boundary conditions, it is rather complicated to obtain analytical solution directly. Considering an inverse 
solution where the vibration mode shape function is assumed as

$$
\psi_{n}(\bar{x})=\sin n \pi \bar{x} \quad(n=1,2,3, \ldots),
$$

which satisfies the boundary conditions in Eq. (13). The substitution of Eq. (14) into Eq. (11b) yields the dimensionless natural frequency as

$$
\omega_{n}=n \pi \sqrt{\frac{\bar{T}+\left(\bar{T} \tau^{2}+1\right) n^{2} \pi^{2}+2 \tau^{2} n^{4} \pi^{4}}{1+n^{2} \pi^{2} \tau^{2}}},
$$

which, in dimensional form, is

$$
\Omega_{n}=n \pi \sqrt{\frac{T L^{4}+n^{2} \pi^{2}\left[T L^{2}\left(e_{0} a\right)^{2}+E I L^{2}\right]+2 n^{4} \pi^{4} E I\left(e_{0} a\right)^{2}}{\rho A L^{4}\left[L^{2}+n^{2} \pi^{2}\left(e_{0} a\right)^{2}\right]}},
$$

where $n=1,2,3, \ldots$ The analytical solutions in Eqs. (15) and (16) can be easily shown to reduce to the classical solutions ${ }^{41,42}$ for vanishing nanoscale $\tau$. Hence, the validity of the inverse solution is established.

Taking the first mode frequency as an example, a comparison of frequency based on nonlocal stress theory and classical vibration theory is shown in Fig. 2 while the first three vibration mode shapes are shown in Fig. 3. The nonlocal nanoscale is observed to significantly affect the vibration frequencies. Apparently, frequencies for a nonlocal nanobeam are significantly higher than the corresponding solutions based on classical vibration.

The effect of $\tau$ on the vibration frequencies is illustrated in Figs. 4-6, respectively. From the figures, it is observed that the free vibration frequencies increase with increasing $\tau$. Hence, the stiffness of nanobeam increases for higher $\tau$ and the rate of increase is particularly marked for higher vibration modes. A possible explanation is that larger nonlocal nanoscale indicates stronger intermolecular interaction constraints and thus higher stiffness.

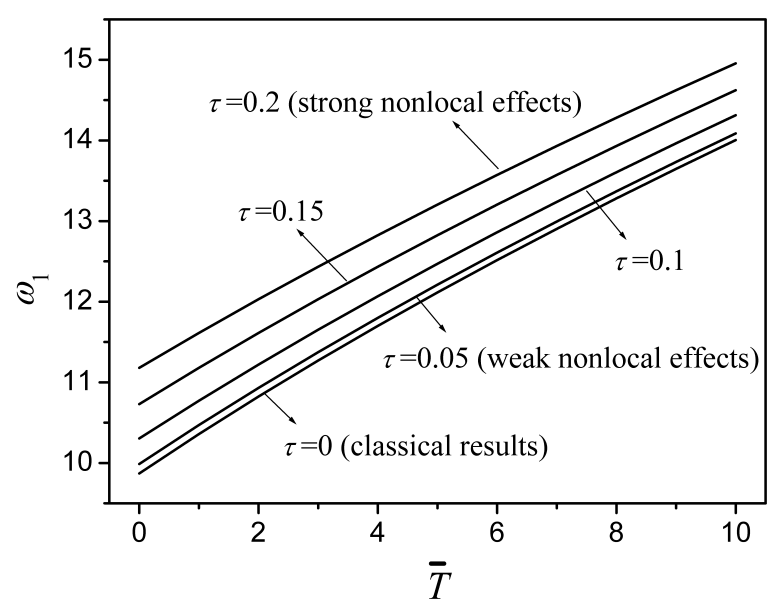

Fig. 2. Comparison of vibration frequency obtained from nonlocal theory and classical theory. 


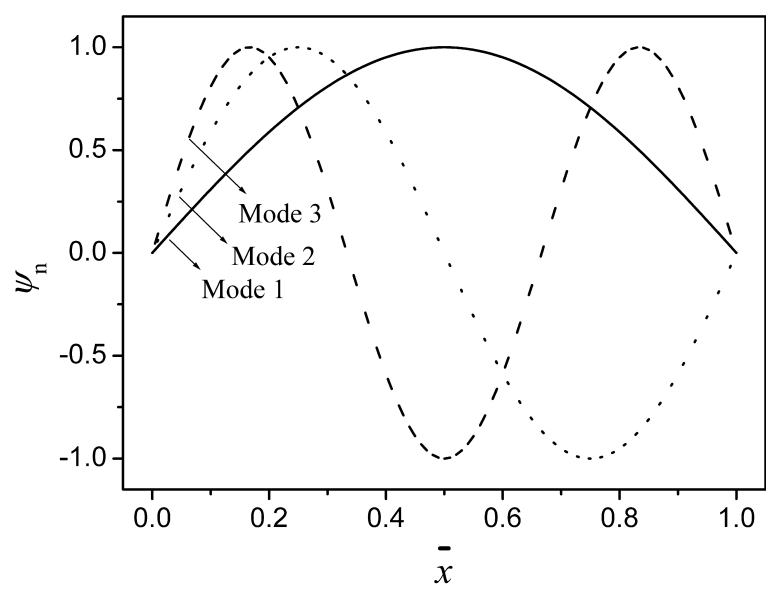

Fig. 3. First three vibration mode shapes.

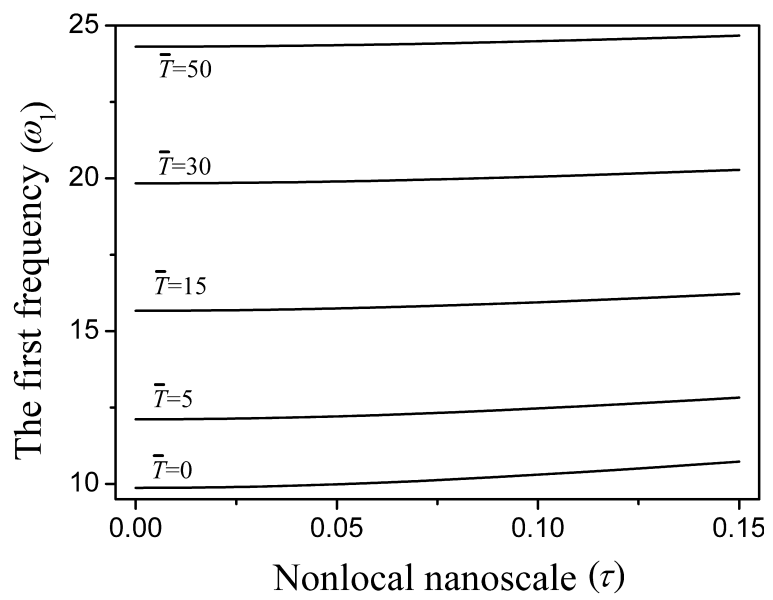

Fig. 4. Effect of nonlocal nanoscale $\tau$ on first mode vibration frequency for increasing dimensionless axial tension $\bar{T}$.

On the other hand, comparison with a nanobeam without axial force shows that the vibration frequencies increase when an initial axial tension is present. Similarly, it can be observed that deflection decreases or stiffness increases due to axial tension. In addition, the vibration frequencies are higher for a larger axial tension. Through a similar analytical procedure, it can be predicted that the vibration frequencies decrease if an axial compression is present and furthermore, the vibration frequencies decrease with increasing compression, which can be readily predicted from Eq. (15) by substituting a negative value for $\bar{T}$. The prediction agrees with the results of classical vibration theory. 


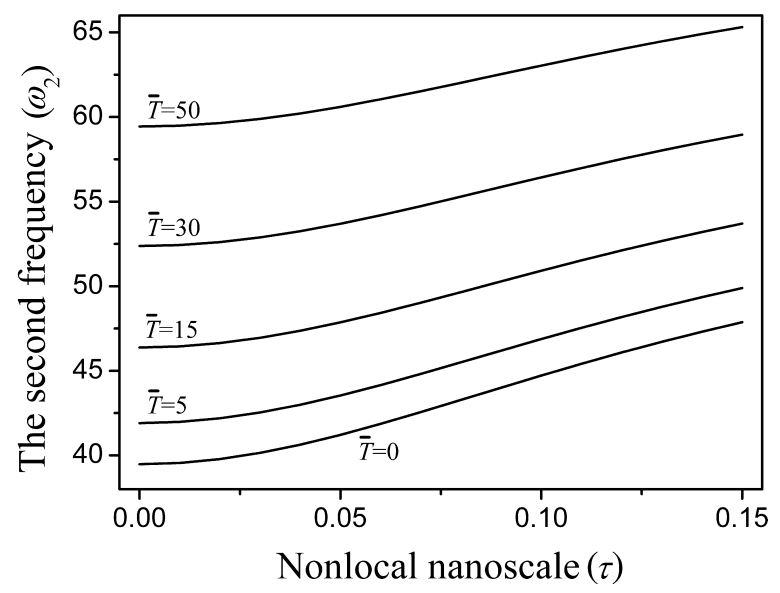

Fig. 5. Effect of nonlocal nanoscale $\tau$ on second mode vibration frequency for increasing dimensionless axial tension $\bar{T}$.

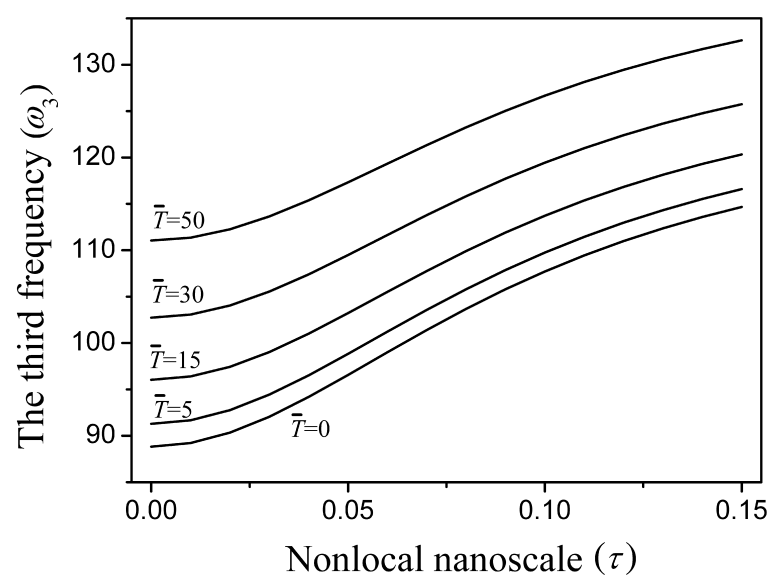

Fig. 6. Effect of nonlocal nanoscale $\tau$ on third mode vibration frequency for increasing dimensionless axial tension $\bar{T}$.

From Eq. (15), the natural frequency of a simply supported nanobeam subjected to an initial axial compression is given by

$$
\omega_{n}=n \pi \sqrt{\frac{-\bar{P}+\left(1-\bar{P} \tau^{2}\right) n^{2} \pi^{2}+2 \tau^{2} n^{4} \pi^{4}}{1+n^{2} \pi^{2} \tau^{2}}},
$$

where $\bar{P}=-\bar{T}$ is defined as a dimensionless compression parameter. The axial compression satisfies

$$
\bar{P} \leq \frac{n^{2} \pi^{2}\left(1+2 \tau^{2} n^{2} \pi^{2}\right)}{1+n^{2} \pi^{2} \tau^{2}}
$$




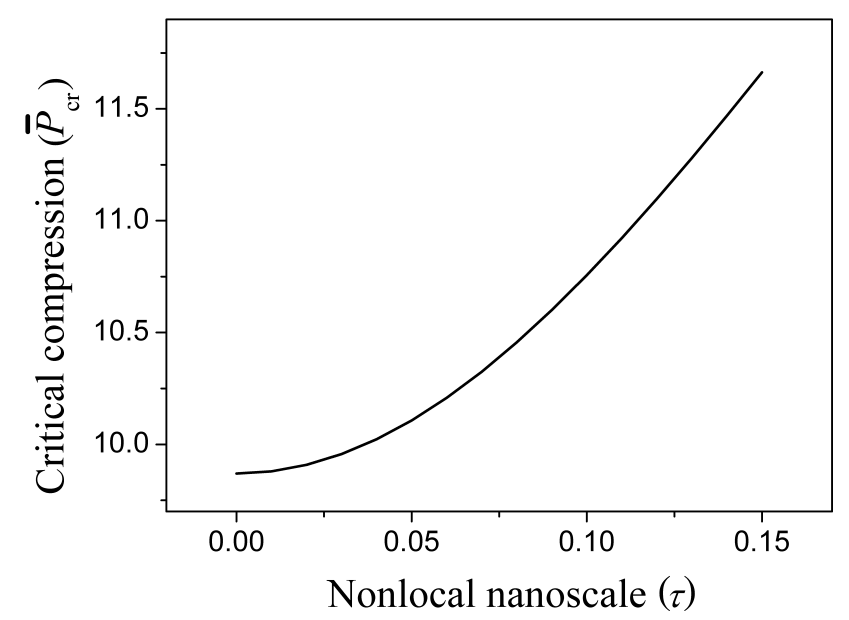

Fig. 7. Critical compression versus the nonlocal nanoscale.

Therefore, the dimensionless critical axial compression for $n=1$ is derived from Eq. (18) as

$$
\bar{P}_{\mathrm{cr}}=\frac{\pi^{2}\left(1+2 \tau^{2} \pi^{2}\right)}{1+\pi^{2} \tau^{2}}=\pi^{2}\left(1+\tau^{2} \pi^{2}+O\left(\tau^{4}\right)\right)
$$

If the nonlocal nanoscale vanishes, the dimensional form of Eq. (19) becomes

$$
P_{\text {cr }}=\frac{\pi^{2} E I}{L^{2}}
$$

which is consistent with the classical buckling theory for columns. Figure 7 shows the relations between the dimensionless critical axial compression and the nonlocal nanoscale. Nonlocal nanoscale enhances the critical axial compression significantly. When $\tau=0.15$, the critical compression increases by $18.2 \%$ with respect to the classical buckling load.

From Eqs. (10), (11a), (14), and (15), the time history mode shapes with respect $\bar{x}$ and $\bar{t}$ can be obtained.

\section{Further Examples with Numerical Solutions}

In this section, a silicon nanobeam is considered in order to demonstrate the difference in nonlocal and classical natural frequencies. From the classical theory, the free vibration frequency of a simply supported beam with an initial axial tension is given by $^{41,42}$

$$
\Omega_{n}^{\mathrm{c}}=n \pi \sqrt{\frac{n^{2} \pi^{2} E I}{\rho A L^{4}}+\frac{T}{\rho A L^{2}}} \quad(n=1,2,3, \ldots),
$$

where the notations have been standardized which those defined in this paper. Note that Eq. (21) can be also derived from Eq. (16) when the nonlocal nanoscale vanishes, 
i.e., $\tau=0$. The deduction of classical solution from the nonlocal solution indirectly confirms the consistency of the new model in the case of vanishing nanoscale effect. This indicates the validity of the model and results presented in this paper again.

\subsection{Example 1}

To make a comparison between Eqs. (16) and (21), an example of a silicon nanobeam is presented here. For a silicon nanobeam with length $L=1 \mu \mathrm{m}$, thickness $H=100 \mathrm{~nm}$, width $B=300 \mathrm{~nm}$, Young's modulus $E=160 \mathrm{GPa}$, mass density $\rho=2400 \mathrm{~kg} / \mathrm{m}^{3}$, and initial axial tension $\bar{T}=20$, the solutions are obtained from Eqs. (16) and (21) and are presented in Fig. 8 for comparison. It shows again that $\tau$ causes vibration frequency to increase. Furthermore, the difference is more distinct for higher modes.

\subsection{Example 2}

Another comparison for the effects of length on the natural frequencies is presented in Table 1. Note that many studies show the mechanical properties, such as Young's modulus is size dependence and the value varies depending on the external

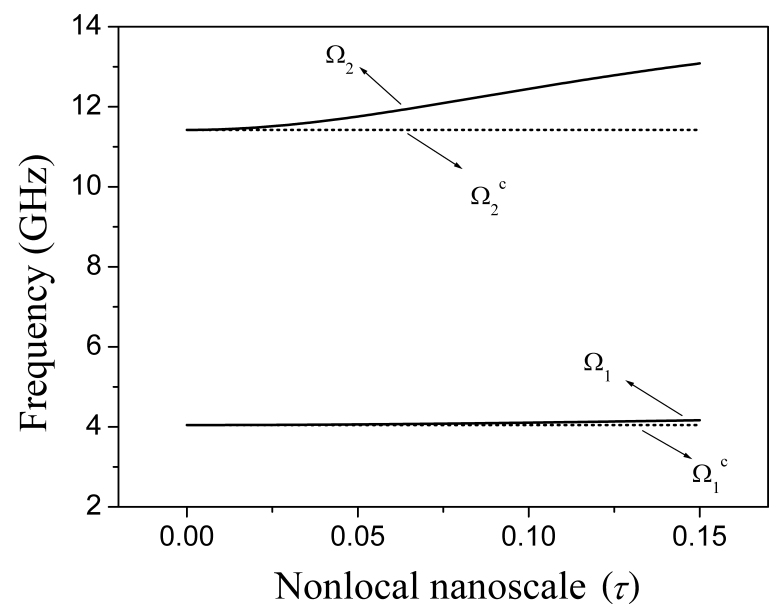

Fig. 8. Comparison between nonlocal and classical frequencies for a silicon nanobeam with varying $\tau$.

Table 1. Comparison between nonlocal and classical frequencies for a silicon nanobeam with varying length.

\begin{tabular}{lrrrrrrrrr}
\hline$\Omega(\mathrm{GHz})$ & \multicolumn{10}{c}{$L(\mathrm{~nm})$} \\
\cline { 2 - 9 } & \multicolumn{1}{c}{80} & 100 & 120 & 140 & 160 & 180 & 200 & 300 & 500 \\
\hline$\Omega_{1}$ & 12.206 & 8.038 & 5.771 & 4.400 & 3.505 & 2.887 & 2.440 & 1.346 & 0.706 \\
$\Omega_{1}^{\mathrm{c}}$ & 12.173 & 8.025 & 5.765 & 4.397 & 3.503 & 2.886 & 2.439 & 1.346 & 0.706 \\
$\Omega_{2}$ & 47.213 & 30.339 & 21.229 & 15.754 & 12.206 & 9.776 & 8.038 & 3.909 & 1.741 \\
$\Omega_{2}^{\mathrm{c}}$ & 46.671 & 30.116 & 21.122 & 15.696 & 12.173 & 9.756 & 8.025 & 3.906 & 1.741 \\
\hline
\end{tabular}


characteristic scale (i.e., the length or thickness of beam), ${ }^{43}$ especially when it is below $20 \mathrm{~nm}$. Here, for a silicon nanobeam with thickness $H=4 \mathrm{~nm}$, width $B=$ $10 \mathrm{~nm}, T=10^{-3} \mu \mathrm{N}$, Young's modulus $E=100 \mathrm{GPa},{ }^{43}, e_{0} a=2.0 \mathrm{~nm}$, and all other parameters are identical to those in Example 1. Table 1 shows a comparison with respect to varying length of the nanobeam.

Both nonlocal and classical theories imply higher frequencies for a very small length of the nanobeam. The natural frequencies increase quickly with decreasing length of the nanobeam, especially for nanobeams with a minute length and nanoscale effect. Equivalently, a nanostructure with nonlocal effect possesses higher vibration frequencies as compared to an identical nanostructure without nonlocal effect. For instance, when the silicon nanobeam decreases from $500 \mathrm{~nm}$ to $200 \mathrm{~nm}$ in length, the first nonlocal and classical frequencies increase by 2.456 times and 2.454 times, respectively, and the difference is not obvious. On the other hand, when the nanobeam decreases from $500 \mathrm{~nm}$ to $80 \mathrm{~nm}$, the first nonlocal and classical frequencies increase by 16.29 times and 16.24 times, respectively. Consequently, the classical theory without nonlocal nanoscale effect undervalues the vibration frequency for nanostructures.

Because the nonlocal effect on vibration frequency is significant, it is indispensable in nanostructural modeling and the classical continuum theory has to be amended. It is also observed that with the increasing length of the nanobeam, the nonlocal and classical continuum theories agree with each other very well. This demonstrates that nonlocal model is consistent with classical model when the structures are at macroscale.

\section{Conclusions}

Considering nonlocal elasticity theory, the free vibration of a nanobeam subjected to an initial axial force is solved via an exact, analytical approach. Exact free vibration frequencies and mode shapes are derived and presented. The nonlocal nanoscale and dimensionless axial force induce significant effects on vibration frequencies. For a simply supported nanobeam, increases in nonlocal effect and axial tensile force cause the vibration frequencies to increase which imply higher nanobeam stiffness. Additionally, an increase in axial compression results in lower natural frequency. Numerical examples show the results are reduced exactly to the classical vibration solutions for vanishing nonlocal nanoscale.

The paper also concludes the governing equation of motion for a nonlocal nanobeam can be formed by replacing the bending moment term in the classical equation of motion with an effective nonlocal bending moment as presented herewith. The result is no longer a fourth-order partial differential equation but an infinite order differential equations with infinitely many higher-order boundary conditions. The latter can be simplified into a sixth-order differential equation with six corresponding higherorder boundary conditions if only the most significant nonlocal term is considered. The nonlocal elasticity approach is very convenient and, to a certain extent, 
indispensable in the continuum approach for modeling, simulation, and analysis of size-dependent nanotubes.

\section{Acknowledgments}

This work was supported by a collaboration scheme from University of Science and Technology of China-City University of Hong Kong Joint Advanced Research Institute and by City University of Hong Kong [Project No. 7002472 (BC)].

\section{References}

1. W. C. Tian and J. Y. Jia, Adhesion research of digital micromirror nano-contact, Micronanoelectronic Technology 39 (2002) 27-30.

2. L. Pellizzari, The development of nanotechnology and nanoelectronics, Elettronica Oggi 320 (2003) 40-44.

3. A. Simpson, Transverse modes and frequencies of beams translating between fixed end supports, J. Mech. Eng. Sci. 15 (1973) 159-164.

4. J. D. Yau, Vibration of parabolic tied-arch beams due to moving loads, Int. J. Struct. Stab. Dyn. 2 (2006) 193-214.

5. B. O. Al-Bedoor and Y. A. Khulief, An approximate analytical solution of beam vibrations during axial motion, Journal of Sound and Vibration 192 (1996) 159-171.

6. L. Q. Chen, J. W. Zu and J. Wu, Principal resonance in transverse nonlinear parametric vibration of an axially accelerating viscoelastic string, Acta Mech. Solida Sinica 20 (2004) $307-316$.

7. H. R. Oz, M. Pakdemirli and E. Ozkaya, Transition behavior from string to beam for an axially accelerating material, Jouranl of Sound Vibration 215 (1998) 571-576.

8. J. G. Guo and Y. P. Zhao, The size-dependent bending elastic properties of nanobeams with surface effects, Nanotechnology 18 (2007) 295701.

9. C. W. Lim and C. M. Wang, Exact variational nonlocal stress modeling with asymptotic higher-order strain gradients for nanobeams, Jouranl of Applied Physics 101 (2007) 054312 .

10. A. C. Eringen, A unified theory of thermomechanical materials, Int. J. Eng. Sci. 4 (1966) 179-202.

11. A. C. Eringen, On differential equations of nonlocal elasticity and solutions of screw dislocation and surface waves, Jouranl of Applied Physics 54 (1983) 4703-4710.

12. E. Kroner, Elasticity theory of materials with long-ranges cohesive forces, Int. J. Solids Struct. 3 (1967) 731-742.

13. J. Peddieson, G. G. Buchanan and R. P. McNitt, Application of nonlocal continuum models to nanotechnology, Int. J. Eng. Sci. 41 (2003) 305-312.

14. Q. Wang and V. K. Varadan, Vibration of carbon nanotubes studied using nonlocal continuum mechanics, Smart Mater. Struct. 15 (2006) 659-666.

15. Q. Wang, V. K. Varadan and S. T. Quek, Small scale effect on elastic buckling of carbon nanotubes with nonlocal continuum models, Physics Letters A 357 (2006) 130-135.

16. J. L. Yu, Progress and applications of solid mechanics considering microstructure, Adv. in Mech. 15 (1985) 82-89.

17. M. H. Zhao, G. J. Cheng, G. N. Liu and Y. P. Shen, The analysis of crack problems with non-local elasticity, Appl. Math. Mech. 20 (1999) 135-143. 
18. P. Lu, H. P. Lee, C. Lu and P. Q. Zhang, Dynamic properties of flexural beams using a nonlocal elasticity model, Journal of Applied Physics 99 (2006) 073510.

19. H. Heireche, A. Tounsi and A. Benzair, Scale effect on wave propagation of double-walled carbon nanotubes with initial axial loading, Nanotechnology 19 (2008) 185703.

20. Y. G. Hu, K. M. Liew, Q. Wang, X. Q. He and B. I. Yakobson, Nonlocal shell model for elastic wave propagation in single- and double-walled carbon nanotubes, J. Mech. Phys. Solids 56 (2008) 3475-3485.

21. L. J. Sudak, Column buckling of multiwalled carbon nanotubes using nonlocal continuum mechanics, Journal of Applied Physics 94 (2003) 7281-7287.

22. Q. Wang, Wave propagation in carbon nanotubes via nonlocal continuum mechanics, Journal of Applied Physics 98 (2005) 124301.

23. C. M. Wang, Y. Y. Zhang, S. S. Ramesh and S. Kitipornchai, Buckling analysis of microand nano-rods/tubes based on nonlocal Timoshenko beam theory, Journal of Physics D: Applied Physics 39 (2006) 3904-3909.

24. Y. Q. Zhang, G. R. Liu and J. S. Wang, Small-scale effects on buckling of multiwalled carbon nanotubes under axial compression, Physical Review. B, Condensed Mater $\mathbf{7 0}$ (2004) 205430.

25. Q. Wang and C. M. Wang, The constitutive relation and small scale parameter of nonlocal continuum mechanics for modeling carbon nanotubes, Nanotechnology 18 (2007) 75702 .

26. C. M. Wang, Y. Y. Zhang and S. Kitipornchai, Vibration of initially stressed micro- and nano-beams, Int. J. Struct. Stab. Dyn. 7 (2007) 555-570.

27. N. Challamel and C. M. Wang, The small length scale effect for a non-local cantilever beam: A paradox solved, Nanotechnolgy 19 (2008) Article No.: 345703.

28. Y. Y. Zhang, C. M. Wang and N. Challamel, Bending and vibration of hybrid nonlocal beams, Journal of Engineering Mechanics, ASCE Journal of Engineering Mechanics 136(5) (2010) 562-574.

29. C. W. Lim, Equilibrium and static deflection for bending of a nonlocal nanobeam, Adv. Vib. Eng. 8 (2009) 277-300.

30. C. W. Lim, Nanoscale for nanobeams based on the theory of nonlocal elastic stress field theory: equilibrium, governing equation and static deflection, Appl. Math. Mech. 31 (2010) $37-54$.

31. C. W. Lim, Nonlocal elasticity theory and application for nanostructures, SciTopics, Elsevier Scirus, March 2010. http://www.scitopics.com/Nonlocal_Elasticity_Theory_ and_Application_for_Nanostructures.html.

32. C. W. Lim, A nanorod (or nanotube) with lower Young's modulus is more stiff? Is not Young's modulus a stiffness indicator? Science in China Series G: Physics, Mechanics 8 Astronomy 53(4) (2010) 712-724.

33. C. W. Lim, C. Li and J. L. Yu, The effects of stiffness strengthening nonlocal stress and axial tension on free vibration of cantilever nanobeams, Interaction and Multiscale Mech. 2(3) (2009) 223-233.

34. C. W. Lim and Y. Yang, New predictions of size-dependent nanoscale based on nonlocal elasticity for wave propagation in carbon nanotubes, Journal Computational and Theoretical Nanoscience 7(6) (2010) 988-995.

35. C. W. Lim and Y. Yang, Wave propagation in carbon nanotubes: Nonlocal elasticity induced stiffness and velocity enhancement effects, Journal of Mechanics of Materials and Structures (in press).

36. C. W. Lim, J. C. Niu and Y. M. Yu, Nonlocal stress theory for buckling instability of nanobeams: New prediction on stiffness strengthening effects of nanoscales, Journal of Computational and Theoretical Nanoscience (in press). 
37. C. W. Lim and Y. M. Yu, Nonlocal elasticity theory for vibration of carbon nanotubes and an analysis of higher-order non-classical boundary conditions (submitted).

38. C. W. Lim, C. Li and J. L. Yu, Dynamic behaviour of axially moving nanobeams based on nonlocal elasticity approach, (submitted).

39. C. Li, C. W. Lim and J. L. Yu, Transverse vibration of pre-tensioned nonlocal nanobeams with internal axial loads (submitted).

40. Y. Yang and C. W. Lim, A new nonlocal cylindrical shell model for axisymmetric wave propagation in carbon nanotubes, (submitted).

41. W. T. Thomson, Theory of Vibration with Applications (Prentice-Hall, Englewood Cliffs, NJ, 1981).

42. Z. Guede and I. Elishakoff, Apparently first closed-form solutions for inhomogeneous vibrating beams under axial loading, Proceedings of Royal Society London A 457 (2001) 623-649.

43. S. H. Park, J. S. Kim, J. H. Park, J. S. Lee, Y. K. Choi and O. M. Kwon, Molecular dynamics study on size-dependent elastic properties of silicon nanocantilevers, Thin Solid Films 492 (2005) 285-289. 